\title{
Ad multos annos!
}

\section{Liebe Leserinnen und Leser,}

Jürgen Weber wird am 4 . November 65 Jahre alt. Anlass genug, kurz innezuhalten und sich den Beitrag eines Hochschullehrers bewusst zu machen, der diese Zeitschrift seit vielen Jahren begleitet hat: in den Jahren 1983 bis 1986 als Schriftleiter und seit 1999 als (Mit-)Herausgeber der Zeitschrift. Dafür möchte ich Jürgen Weber - auch im Namen von Verlag und Redaktion unserer Zeitschrift - Danke sagen und gleichzeitig der Hoffnung Ausdruck verleihen, dass er sich noch viele Jahre in den Dienst der Controlling \& Management Review stellt. Auch und gerade weil es im Alltag viel zu oft untergeht: Danke, Jürgen!

Lassen Sie uns zurückschauen: Schon immer ermöglicht die Controlling \& Management Review den Austausch zwischen Finanzvorständen und Controllern und ist gleichzeitig eine ganz zentrale Plattform für den Dialog zwischen Wissenschaft und Praxis. Dennoch hat sich die Zeitschrift auch über die Zeit verändert, wie sich nicht zuletzt im Namen zeigt. Die Zeitschrift, die Jürgen Weber 1999 als Herausgeber übernommen hat, hieß noch „Kostenrechnungspraxis“ und wurde dann in „Zeitschrift für Controlling \& Management“ und schließlich in „Controlling \& Management Review“ umbenannt. Die Kostenrechnungspraxis der 1980er und 1990er Jahre war - nomen est omen - noch stark von der Kostenrechnung und der Idee einer wirklichkeitsgetreuen Abbildung des wirtschaftlichen Geschehens beseelt, ganz in der Tradition von Paul Riebel und Wolfgang Männel. Unter der Herausgeberschaft von Jürgen Weber hat sich das Schritt für Schritt verändert. Zum einen in der Breite der behandelten Themenfelder, die heute weit über die Kostenrechnung hinausgehen und mit den Rubriken Unternehmenssteuerung, Accounting \& Reporting, Controller \& Manager sowie Information \& Technologie das ganze Tätigkeitsspektrum von Controllern abdecken. Zum anderen in der zugrunde liegenden Perspek- tive: Als Herausgeber gehen wir heute davon aus, dass die Welt sozial konstruiert ist, es also die eine Wirklichkeit nicht gibt. Wir beobachten, dass Menschen potenziell opportunistisch handeln, nach Legitimität streben und nicht unerheblich kognitiv begrenzt sind. Und wir glauben, dass diese menschlichen Eigen-

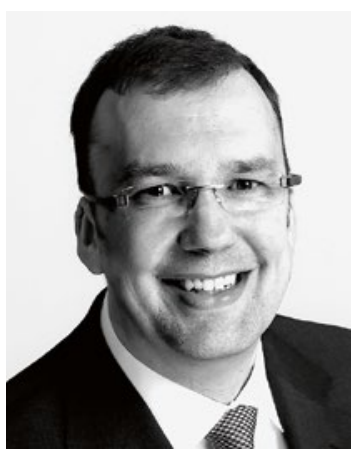

Utz Schäffer schaften das Controlling ganz maßgeblich prägen und wir also genau hier ansetzen sollten, wenn es darum geht, Controlling zu gestalten und zu verändern. Wenn Sie wollen, hat eine verhaltensorientierte Perspektive die traditionelle Sicht eines Ingenieurs ersetzt.

Beide Veränderungen der Zeitschrift gehen ganz wesentlich auf das Wirken von Jürgen Weber zurück und sind im Kontext der Digitalisierung des Controllings relevanter denn je. Daher möchten wir mit dem vorliegenden Heft die Idee der Verhaltensorientierung des Controllings im Spiegel ausgewählter Beiträge von Jürgen Weber aus den Jahren 1994, 2003 und 2007 vertiefen. Sie werden sehen, dass alle drei Aufsätze nichts an Aktualität und Relevanz eingebüßt haben. Daneben frage ich Jürgen Weber im Interview, was er in mehr als 30 Jahren als Hochschullehrer gelernt hat. Lassen Sie sich überraschen!

Viel Spaß bei der Lektüre wünscht Ihnen

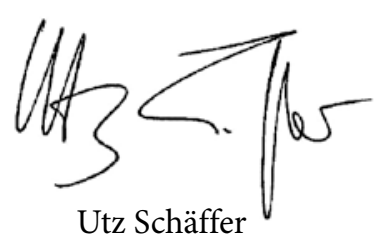

